\section{Regards sur l'économie allemande}

Bulletin économique du CIRAC

$110 \mid 2013$

Varia

\title{
R\&D : la coopération franco-allemande dans la pratique
}

Elisabeth Baier, Andrea Zenker et Volker Tippmann

Traducteur : Isabelle Bourgeois

CpenEdition

Journals

Édition électronique

URL : http://journals.openedition.org/rea/4591

DOI : 10.4000/rea.4591

ISSN : 1965-0787

Éditeur

CIRAC

Édition imprimée

Date de publication : 15 octobre 2013

Pagination : 27-36

ISSN : 1156-8992

Référence électronique

Elisabeth Baier, Andrea Zenker et Volker Tippmann, « R\&D : la coopération franco-allemande dans la pratique ", Regards sur l'économie allemande [En ligne], 110 | octobre 2013, mis en ligne le 01 octobre 2015, consulté le 01 mai 2019. URL : http://journals.openedition.org/rea/4591 ; DOI : 10.4000/ rea.4591 


\section{R\&D : la coopération franco- allemande dans la pratique}

\author{
E. Baier, A. Zenker, V. Tippmann
}

L'analyse que nous présentons ici est le fruit de l'expérience de la coopération franco-allemande en matière de recherche. Elle reflète le vécu des chercheurs, directeurs de recherche et responsables de programmes de recherche bilatéraux interrogés par l'Institut Fraunhofer ISI dans le cadre de l'accompagnement des programmes bilatéraux entre instituts Fraunhofer et Carnot (Zenker et al., 2013a), mandaté par le ministère fédéral de la Recherche. La principale conclusion en est que le succès d'une coopération bilatérale en matière de recherche dépend d'une manière essentielle de facteurs individuels, institutionnels et politiques. En effet, la difficulté que pose la gestion d'un financement conjoint, conjuguée aux différences culturelles, exige de la part des membres des équipes de recherche impliquées des capacités organisationnelles et managériales prononcées, ainsi qu'une grande ouverture à l'autre, doublée d'une profonde tolérance, lorsqu'ils développent une approche commune du projet pour le réaliser au quotidien. Or ce qui semble à première vue un 'surcroît de travail' se révèle en réalité très fructueux parce qu'il élargit l'accès à des infrastructures et à des savoirs existants, mène à une compréhension plus approfondie et plus intime des enjeux de l'interculturel, et favorise l'accès aux réseaux personnels des participants comme des marchés dans le pays partenaire.

Après avoir rappelé l'importance d'une intensification de la coopération transfrontières dans le domaine de la recherche, nous expliciterons les spécificités inhérentes à la coopération franco-allemande, telles qu'elles sont apparues dans l'expérience concrète des coopérations étudiées. Enfin, dans une analyse intégrant les dimensions personnelles, institutionnelles et politiques ou sociétales, nous en présenterons les facteurs de succès comme les handicaps, et nous en tirerons quelques conclusions pour optimiser la coopération bilatérale dans le domaine de la recherche.

On observe, ces dernières années, une intensification générale de l'internationalisation de la recherche tant industrielle que financée par les pouvoirs publics. C'est ce que révèle la hausse des publications scientifiques co-signées par des auteurs issus de différents pays, la progression de la mobilité transfrontières des chercheurs impliqués dans la R\&D comme la recherche fondamentale, ainsi que l'approche de plus en plus transnationale ou globalisée des modes de réflexion et de travail, y compris au niveau institutionnel. Cette dernière évolution s'observe très nettement à travers la multiplication de stratégies afférentes développées par les organisations finançant la recherche, les grands réseaux d'instituts de recherche, ainsi que par le politique.

Les motifs de cette internationalisation sont multiples. L'un des principaux est la possibilité qu'ouvre une coopération transfrontières d'accéder à des compétences et sources du savoir plus larges ; ce motif est déterminant dans le cas où le savoir résidant hors des frontières apporte un complément aux savoirs nationaux. Les coopérations internationales permettent également de réunir plus facilement les ressources nécessaires, ce qui est déterminant surtout dans le cas des grands projets de recherche fondamentale dont les besoins en matière d'infrastructures, de financement et de ressources humaines dépasseraient de loin les capacités d'un seul pays. L'exemple le plus parlant et le plus réputé est le Conseil Européen pour la Recherche Nucléaire (CERN), le plus grand labora-

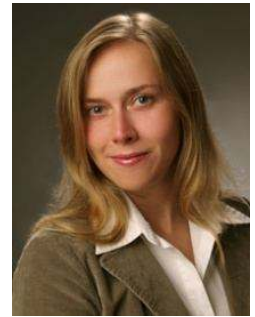

Elisabeth Baier, économiste, Responsable études de marché, PTV Group

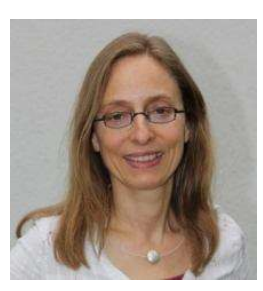

Andrea Zenker, Responsable de projets, Fraunhofer ISI, Karlsruhe

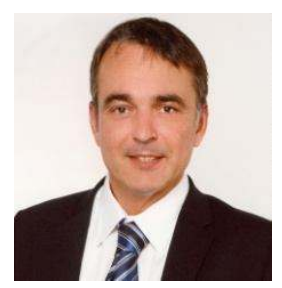

Volker Tippmann, ingénieur,

Département Planification de la Recherche, Fraunhofer Gesellschaft, Munich

Tendance à l'internationalisation de la recherche 
toire de physique nucléaire du monde dont les 20 Etats membres se répartissent les 850 millions $€$ de budget annuel. Un autre motif poussant à la coopération internationale est la volonté d'accroître par-delà les frontières du propre pays le rayonnement scientifique et d'en développer ainsi la réputation mondiale. Enfin, l'intensification de telles coopérations facilite également l'identification précoce de nouvelles tendances scientifiques ou technologiques, ce qui a des effets bénéfiques sur la qualification des chercheurs. L'accès à des paradigmes de recherche nouveaux ou différents incite en effet à développer de nouvelles approches en matière de réflexion ou de quête de solutions qui contribuent à leur tour à générer des avantages compétitifs.

Malgré tous les avantages qu'elle présente, l'intensification de la recherche transfrontières comporte certains risques, en particulier ceux d'une fuite incontrôlée des savoirs, d'un brain drain via une tendance durable à l'émigration d'actifs hautement qualifiés, s'accompagnant d'une hausse des coûts. Pourtant, en règle générale, on estime que les avantages sont plus importants que les risques encourus, ce qui explique cette intensification de la coopération transfrontières en matière de recherche.

\section{Coopération franco-allemande : un état des lieux et trois cas de figure relevés}

France et Allemagne, partenaires privilégiés, ...
... malgré d'importantes différences
Dans la catégorie des coopérations internationales en matière de recherche publique, les laboratoires français figurent parmi les partenaires principaux de leurs homologues allemands et vice versa. Une analyse des partenaires des instituts de recherche allemands a ainsi révélé que la France vient en seconde position après les Etats-Unis (Ebersberger et al., 2007). Dans le domaine des programmes de coopération universitaire, la France vient en troisième position, immédiatement après les Etats-Unis et le Royaume uni (Ebersberger et al., 2007). Ce classement est confirmé par diverses études plus récentes qui le font apparaître également en ce qui concerne les publications scientifiques conjointes: les chercheurs français sont le troisième partenaire de leurs homologues allemands, après les Américains et les Britanniques, tandis que les Allemands sont le premier partenaire des Français (Michels et al., 2013).

Malgré les importantes différences culturelles entre la France et l'Allemagne dans la mise en œuvre de programme de recherche et de R\&D (notamment en ce qui concerne la perception du temps ou du rôle du management ; voir Barmeyer/Davoine, 2013), ni le classement des partenaires privilégiés ni l'intensité de la coopération n'ont de quoi étonner. Depuis longtemps en effet, la France et l'Allemagne sont des partenaires privilégiés en matière d'échanges commerciaux (import et export). Les organismes et laboratoires de recherche de nos deux pays ont une haute réputation, ce qui incite aux échanges dans la quête de l'excellence. Enfin, depuis cinquante ans, la coopération franco-allemande est une déterminante de l'action politique de nos deux pays ; le Traité de l'Elysée signé en 1963 par de Gaulle et Adenauer a intensifié la coopération francoallemande et abouti à une coordination politique dans divers domaines.

Pourtant, les systèmes français et allemand de recherche et d'innovation présentent de grandes différences (Zenker et al., 2013b) dans leurs caractéristiques et leurs traditions. Alors que le système français est plutôt centralisé, celui de l'Allemagne fédérale est largement décentralisé. Dans les deux pays, universités et centres de recherche extra-universitaires sont certes des acteurs majeurs de la recherche; mais l'étroitesse des liens entre recherche et enseignement n'est pas la même, elle obéit à des traditions différentes (Condorcet/ Humboldt ; voir Jeltsch, 2013). Néanmoins, on observe depuis plusieurs années des évolutions parallèles : ainsi, par exemple, les grands programmes de recherche ont gagné en importance dans les deux pays durant les années 1980 ; de même, les stratégies et initiatives d'excellence nationales y ont été élaborées et lancées à peu près au même moment. 
Dans le domaine de la recherche scientifique, la mise en œuvre et le développement continu de la coopération bilatérale s'effectuent au niveau politique, les consultations entre différents ministères et organismes de recherche débouchant sur des programmes de recherche. Parmi les accords conclus au cours des dernières années, il faut également relever les programmes adoptés en matière de recherche appliquée comme le Programme Inter Carnot-Fraunhofer (PICF ; Tippmann/Buoncuore 2013), ou encore l'harmonisation de programmes nationaux de la Deutsche Forschungsgemeinschaft (DFG) et de l'Agence Nationale de le Recherche (ANR) pour financer des programmes de recherche fondamentale. Celle-ci, qui commence à porter ses premiers fruits après une longue phase de conception, marque une étape décisive dans le développement de la coopération dans ce domaine (Haag, 2013).

Au total, on constate un large éventail de coopérations bilatérales en matière de recherche. II s'étend de l'échelon institutionnel jusqu'à celui des individus : instituts de recherche binationaux (Kaufmann-Spachtholz et al., 2013), harmonisation de programmes de recherche au niveau institutionnel (Haag, 2013 ; Tippmann/Buoncuore, 2013), ouverture de programmes nationaux à des partenariats (Ballouk, 2013 ; Tippmann, 2013), programmes réalisés par des équipes binationales procédant à l'échange de chercheurs ou création d'équipes pérennes (Weg-Remers et al., 2013). S'y ajoutent les coopérations en matière de recherche proche du marché auxquelles se livrent les entreprises et qui sont par exemple financées au sein d'incubateurs transfrontières (Damiani, 2013).

Dans le cadre de l'étude sur différents cas choisis de coopérations francoallemandes que nous avons menée sur la base d'entretiens avec des chercheurs, des directeurs de laboratoires et des directeurs de programmes de recherche, il est apparu que les formes concrètes que prennent ces coopérations sont très variées. Leur succès dépend en effet, dans le détail, de nombreux facteurs dont certains peuvent être partiellement définis au niveau individuel, mais dont d'autres sont déterminés par l'environnement réservé à ces activités. Par ailleurs, d'autres facteurs encore sont perçus comme favorables ou à l'inverse handicapants par les directeurs de programmes concernés.

Les trois cas que nous présentons ci-dessous à titre d'exemple sont caractéristiques de l'expérience vécue par les scientifiques impliqués dans ces coopérations, en ce sens qu'ils révèlent la complexité de l'approche et la multiplicité des différences dans la perception individuelle de ces coopérations comme des pratiques et routines tant institutionnelles que sociétales.
Un large éventail de coopérations bilatérales...

... et des approches multiples

Trois cas de figure révélateurs

Cas $n^{\circ} 1$ : L'importance de la flexibilité, de la mobilité, de la liberté de la recherche et de la compétence en matière interculturelle pour le succès de programmes de recherche bilatéraux - l'expérience recueillie dans un programme de recherche fondamentale bénéficiant du soutien de l'ANR-DFG (Savoy/Nerlich, 2013).

Facteurs de succès :

- Les facteurs clés sont le contact personnel et de fréquentes rencontres. La qualité de l'échange que permet la présence face à face favorise l'implication intense dans le travail - y compris dans la manière de procéder - et, partant, la genèse d'une approche conjointe dans le développement du projet. C'est la raison pour laquelle sont organisés de fréquents workshops s'étendant souvent sur plusieurs journées. Or cela implique l'existence d'un budget voyages d'un montant suffisant - cet aspect devrait être pris en considération en amont, dès la phase de préparation du dossier de candidature.

- Un avantage des programmes ANR-DFG réside dans la grande souplesse dont disposent les équipes de chercheurs dans l'implémentation du programme. Ainsi, par exemple, les institutions finançant le programme se montrent ouverts aux suggestions de redéploiement des moyens dès lors que ceux-ci sont argumentés et judicieux.

- L'indépendance en matière de contenu de la recherche est un facteur de réussite très important pour les programmes bilatéraux - tout particulièrement dans le domaine de la recherche fondamentale ou sans objectif prédéfini.

Les défis à relever :

- D'une manière générale, tant en France qu'en Allemagne, il y a peu d'étudiants qui connaissent bien la langue et la culture du pays partenaire, ce qui rend difficile le recrutement de collaborateurs (faible nombre de candidatures). Pour être couronnés de succès, les programmes de recherche menés en coopération devraient donc prévoir aussi le financement en partie de l'apprentissage de la langue du partenaire.

- Les grandes différences entre les systèmes universitaires et de formation professionnelle ne facilitent pas la coopération bilatérale ni son intégration dans l'enseignement et la recherche académique. Dès leur conception, ces programmes devraient donc prévoir une certaine souplesse permettant de mettre à profit ces différences systémiques et de les considérer comme un défi à relever. 
Cas $n^{\circ} 2$ : Coopération franco-allemande dans le domaine de la recherche sur le cancer - l'expérience recueillie lors de la mise en place d'une unité de recherche bilatérale (Weg-Remers et al., 2013).

Facteurs de succès :

- L'expérience personnelle de l'interculturel qu'ont les collaborateurs de cette unité s'est révélée fondamentale pour surmonter les difficultés qui peuvent naître des différences culturelles.

- La maîtrise de l'anglais a une importance particulière, la langue principale dans cette unité de recherche étant l'anglais. - Les contacts personnels et l'insertion dans les réseaux spécialisés sont d'une grande importance, surtout en France où les contacts informels sont décisifs.

Les défis à relever :

- Le financement de projets de recherche bilatéraux est souvent difficile à réaliser, étant donné l'absence de programmes bilatéraux afférents et les différences foncières entre les structures organisationnelles comme le financement des institutions en charge de la recherche en France et en Allemagne.

-Du fait qu'il n'existe pas de programme bilatéral de financement de la recherche, chaque projet de recherche est soumis à une procédure d'autorisation, à la fois en France et en Allemagne, ce qui complique et, surtout, allonge inutilement la phase de coordination.

Cas $n^{\circ} 3$ : Coopérations bilatérales en recherche appliquée : du développement d'un prototype jusqu'à la mise sur le marché - l'expérience du Programme Inter Carnot-Fraunhofer PICF (Dimroth, 2013).

Facteurs de succès :

- Les deux organismes de recherche concernés avaient la volonté déclarée de coopérer, et le programme de recherche bilatéral présentait une opportunité unique pour établir une coopération stratégique visant l'excellence. De plus, ce programme offrait une structure de financement stable durant la phase de recherche et de développement.

- Il a été possible de conjuguer et d'optimiser infrastructures et compétences complémentaires, tout en respectant la protection des savoirs et savoir-faire propres à chacun des partenaires. L'association précoce de partenaires industriels garantissait l'accès au marché.

-Structures de communication efficientes et effet stabilisateur de 'personnes clés' sur les relations interpersonnelles ont joué un rôle déterminant.

-Les partenaires mènent conjointement la recherche de nouvelles options stratégiques comme de débouchés.

Les défis à relever :

- Financement dans la phase du développement jusqu'à la commercialisation : le financement prévu dans le cadre du programme bilatéral couvrait la phase de R\&D, mais non pas celle précédant la commercialisation; il convient ici de trouver un financement-relais.

-Plus généralement, la stabilité des conditions de financement jusqu'à la phase de commercialisation est indispensable ; ces conditions devraient être modulables selon les spécificités de chaque projet.

Ces trois cas de figure montrent que les principales sources de défis dans le cadre de la coopération franco-allemande dans le domaine de la recherche sont les différences culturelles et, bien plus souvent encore, le manque d'harmonisation des structures de financement. Quant aux facteurs de succès, ils s'observent le plus généralement dans la personnalité et l'approche individuelle des chercheurs, mais n'en sont pas moins déterminés par les structures organisationnelles respectives.

\section{Les facteurs de succès: personnes, structures et éléments contextuels}

Trois niveaux : Les bilans d'expérience recueillis auprès des participants à tous les projets de coopération franco-allemande analysés dans l'ouvrage cité font apparaître que les facteurs déterminants pour la réussite de tels projets sont multiples. Ils interviennent à trois niveaux : personnel, c'est-à-dire à celui de l'équipe menant à bien le projet (organisation, management, par exemple), institutionnel et enfin, à l'échelon supérieur, politique (voir le graphique ci-après). A chacun de ces niveaux apparaissent des défis susceptibles de menacer la poursuite de la coopération s'ils ne sont pas relevés. Et, il convient de le souligner, ces trois niveaux interfèrent et ne peuvent donc être considérés isolément.

$1 /$ les personnes, $\ldots$ Ceux parmi les chercheurs qui participent à des programmes bilatéraux couronnés de succès jugent souvent que cette coopération est positive et enrichissante. Ils apprécient l'ouverture qu'elle apporte à leur propre horizon, qu'il s'agisse de leur spécialité ou de la compétence à l'interculturel qu'elle leur permet d'acquérir et qui accroît leur qualification pour des programmes de recherche 
transnationaux ultérieurs. En règle générale, les chercheurs impliqués dans ce type de programmes sont en effet confrontés dans la pratique aux différences existant dans les cultures et modes de travail des deux partenaires, notamment en ce qui concerne les structures décisionnelles et les modes de communication. Or ces derniers sont déterminants pour le bon déroulement du projet ; et à presque tous les stades du travail en équipe transnationale, il est fondamental de comprendre quels sont les processus qui, dans la culture de l'autre, aboutissent à des prises de décision et quelles sont les personnes impliquées.

\section{Les trois niveaux de facteurs déterminants pour les coopérations bilatérales}

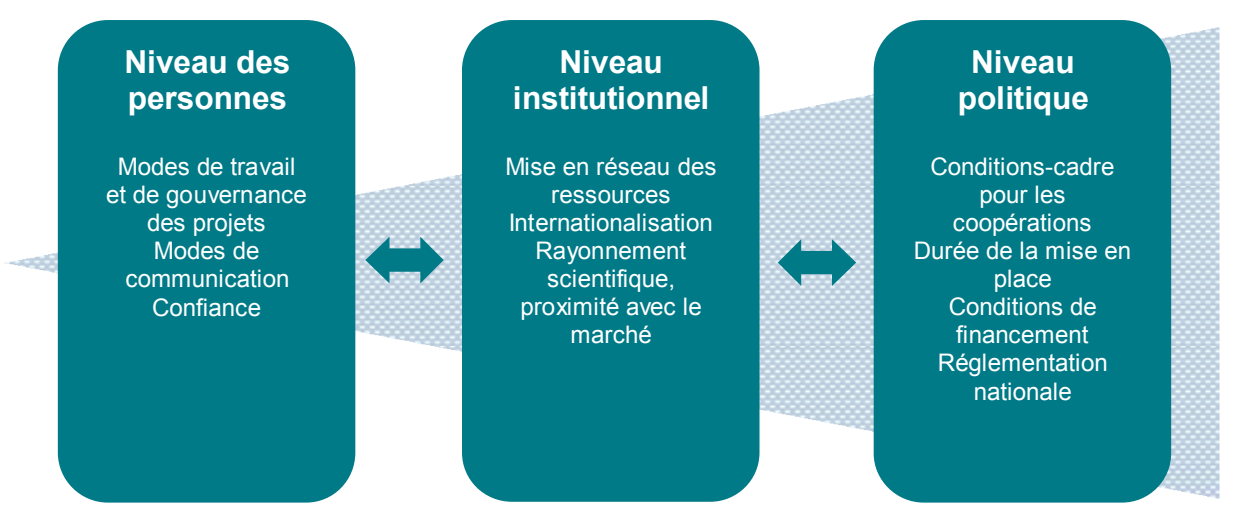

Source : auteurs de l'analyse.

Outre la prise de conscience de ces différences, c'est la sensibilité aux particularités respectives et la capacité à les prendre en compte à bon escient qui apporte une contribution essentielle au succès de ces projets et programmes. Les participants sont ainsi mieux armés pour prendre des décisions lorsqu'ils savent qu'en Allemagne, une décision est habituellement l'aboutissement d'un processus formel et que, une fois adoptée, celle-ci constitue la base sur laquelle sera prise toute décision ultérieure, alors qu'en France, il est d'usage de remettre à plat en permanence toute décision. Les modes de communication aussi diffèrent : en Allemagne, ils sont plus formalisés et se concentrent sur le 'cœur du sujet', alors qu'en France, il importe avant toute chose de mieux faire connaissance personnellement avec les (futurs) collègues avant d'aborder les questions liées au travail.

C'est cela qui explique les réponses qui peuvent sembler étonnantes au premier abord des scientifiques allemands quand on leur demande quelles différences culturelles ils ont constatées : ils évoquent alors souvent en premier lieu la pratique des réunions informelles. En France en effet, les repas pris en commun sont souvent le moyen de faire connaissance, de s'échanger sur la vie privée comme sur le travail ; ils sont l'occasion de construire des liens de confiance mutuelle, et constituent ainsi la base d'une bonne entente au travail. En Allemagne à l'inverse, l'habitude veut qu'on sépare plus nettement questions privées et professionnelles. Quand ces différences dans les pratiques de communication sont connues, ce mode de construction de la confiance mutuelle est non seulement reconnu, mais prisé par les deux parties qui vont en cumuler les effets en multipliant les rencontres régulières ou les workshops, etc.

Si la maîtrise de la langue du partenaire n'est pas une condition sine qua non, elle n'en permet pas moins d'accélérer le processus qui permet de faire connaissance pour construire des liens de confiance, et de faciliter la communication au sein de l'équipe. Si en revanche, cette sensibilité aux différences culturelles et contextuelles n'existe pas et si les chercheurs impliqués partent du principe que, dans les autres pays, les équipes procèdent et agissent de la même manière que chez eux, alors peuvent se produire des situations critiques.
... leur capacité à gérer les différences culturelles...

... et leur maîtrise de la langue du partenaire 
2/ le niveau institutionnel...

... et le soutien qu'il apporte au projet

3/ le niveau politique...

... et sa capacité à innover...
L'incompréhension mutuelle peut alors provoquer des retards dans le déroulement du projet et même la non-réalisation de certains objectifs.

De tels processus d'apprentissage s'observent également au niveau des organismes de recherche, par exemple dans l'organisation de projets transnationaux ; ils peuvent être utilement mis à profit dans tous les projets ultérieurs de coopération transfrontières, de même qu'à l'échelon européen. Par ailleurs, ces coopérations présentent de multiples avantages stratégiques: outre qu'elles permettent de mettre en réseau compétences, méthodes, infrastructures et moyens financiers, elles favorisent réputation et rayonnement scientifiques, accès aux réseaux du partenaire, ainsi qu'à son marché (ce qui est fondamental dans le cas de la recherche orientée vers l'industrie). Enfin, la coopération bilatérale apporte une contribution importante à la mise en œuvre des stratégies d'internationalisation que mènent les organismes ou instituts de recherche des deux pays. Dans cette perspective, les organismes de recherche jouent un rôle important dans la réussite des travaux de recherche transnationaux en créant un environnement porteur pour les projets conjoints.

Dans la pratique des coopérations existantes, on relève un certain nombre de facteurs de succès. Le plus important est le soutien apporté par l'organisme de recherche ou le laboratoire qui accueille les équipes conjointes. L'expérience a montré également que le contexte et les conditions de départ sont particulièrement porteurs quand les projets sont soutenus activement par la direction (direction de l'équipe, du laboratoire ou de l'institut) des organismes concernés. Enfin, les coopérations connaissent une évolution particulièrement positive lorsque des personnes clés (surtout si elles sont de surcroît habilitées à prendre des décisions) s'investissent dans le montage du projet de coopération ou les accompagent d'une manière constructive (voire y sont impliquées), ou encore exercent une fonction de médiateur en cas de conflit. En règle générale, ces personnes ont une connaissance intime du pays partenaire, de sa culture, de son système de recherche et de ses structures institutionnelles.

Le troisième niveau jouant un rôle déterminant dans le succès des coopérations bilatérales dans le domaine de la recherche est celui du politique, les pouvoirs publics définissant le contexte dans lequel évoluent les équipes transnationales, en l'occurrence en concevant des programmes de recherche porteurs. Or l'harmonisation requise des programmes nationaux de recherche doit relever de nombreux défis pour que l'organisation et le déroulement des coopérations bilatérales soient couronnés de succès. L'expérience a montré en effet que si la coordination ou l'harmonisation se révèlent insuffisantes, on aboutit dans la réalité à la coexistence de deux projets nationaux indépendants qui se déroulent en parallèle dans le meilleur des cas. Pourtant, si on veut impliquer et valoriser au mieux les compétences des partenaires respectifs, il est indispensable de procéder à un partage concerté des tâches, et à relier entre eux en les articulant les différents aspects de la réalisation du projet comme les résultats de la recherche - et ce, de la phase de préparation du dossier de candidature jusqu'à la valorisation des résultats ou la commercialisation.

Une connaissance intime de l'organisation des programmes, des structures et processus décisionnels dans les deux pays à la fois est fondamentale pour mettre en cohérence les différents programmes de recherche et en unifier les procédures. Sont également indispensables une grande ouverture d'esprit et la capacité individuelle à déroger aux usages auxquels on est habitué pour bâtir un consensus conciliant la politique de recherche et les pratiques en matière de financement de la recherche des deux pays partenaires. Car si on veut mener à la réussite des projets bilatéraux, il faut les concevoir comme un ensemble cohérent, ce qui exige un cadre commun pour leur financement. Dans le cas des coopérations franco-allemandes, il s'agit ainsi de concilier entre elles les différences dans la planification du temps, les procédures de dépôt des candidatures, de l'évaluation et du financement des projets de recherche. 
Quand, par exemple, les partenaires français prévoient la participation de doctorants au projet, ils sont dans une certaine mesure liés par la durée des différentes formes de soutien financier aux thésards en France, et éventuellement par la durée des contrats liant les doctorants à leur université ; cette situation peut mener à une divergence des cycles dans la réalisation du projet entre les deux partenaires. De même, les pratiques en matière de candidature diffèrent. Les procédures prévoient par exemple une seule ou deux étapes : il s'agit soit du dépôt d'un dossier complet (c'est le cas en France et partiellement en Allemagne), soit à l'inverse du dépôt d'un avant-projet, soumis à évaluation, puis du dépôt d'un dossier complet (c'est le cas partiellement en Allemagne). Chacun de ces modes de gestion a des répercussions immédiates sur la durée totale de la procédure de candidature comme de l'évaluation. Sans une procédure unifiée, le lancement conjoint d'un projet n'est guère possible, d'autant plus qu'un projet bilatéral requiert le dépôt d'un dossier de candidature conjoint, répondant aux mêmes conditions et soumis à une procédure d'évaluation unique effectuée par le même groupe d'experts. Enfin, il faut mieux prendre en considération les différents modes de financement en usage dans les organisations ou instituts de recherche respectifs : les instituts disposant de peu de fonds propres n'ont pas la même approche de la planification financière d'un projet que ceux disposant d'importants moyens propres qui leur permettent de couvrir les dépenses.

$\mathrm{Au}$ vu de tous ces aspects, l'insertion de ces projets dans des accords bilatéraux et des déclarations d'intention communes, des memorandum of understanding ou autres, se révèle favorable à la coopération. La prise en compte de ces différents aspects s'accompagnant d'un important processus d'apprentissage de la part des organismes gestionnaires des programmes, elle se traduit certes par une longue phase de préparation avant d'aboutir finalement à une harmonisation des approches respectives. Mais à terme, cela a des effets bénéfiques au niveau où sont déposés les dossiers de candidature et mis en œuvre les projets, étant donné que les accords bilatéraux permettent ainsi de réduire souvent considérablement la durée de la phase de préparation de chaque programme ou projet.

Pour résumer, une étroite coopération comme des échanges réguliers entre les sociétés ou organismes de financement de la recherche contribuent à la simplification administrative et facilitent la réalisation de coopérations bilatérales. II s'agit moins, dans l'ensemble, de chercher à gommer totalement les différences existantes, mais bien plutôt de les identifier et de les gérer dans l'intérêt de toutes les parties prenantes. Ce n'est qu'ainsi que le succès des coopérations en matière de recherche pourra être plus que la somme de l'apport de chacune des structures impliquées, et que les enseignements tirés de ces différences seront profitables à tous les niveaux concernés.

\section{Un cas d'école : le Programme Inter Carnot-Fraunhofer (PICF)}

Le programme franco-allemand Inter Carnot-Fraunhofer (PICF) est à cet égard riche d'enseignements quant au montage d'un programme de soutien bilatéral à la recherche et aux facteurs de réussite liés. A la différence des cas présentés ci-dessus, et qui retraçaient - dans la perspective individuelle de chacun des partenaires impliqués - l'expérience faite dans le cadre de projets de coopération isolés, l'exemple du PICF permet d'analyser le cas d'un programme de soutien bilatéral sous l'angle des enseignements tirés conjointement au niveau des institutions et de la coordination des programmes; les expériences réciproques sont ici agrégées.

Lors du deuxième forum franco-allemand de la recherche qui s'est tenu à Potsdam en 2005, les ministres français et allemand de la recherche avaient conclu un accord visant à approfondir la coopération en matière de politique d'innovation et de recherche. C'est ainsi qu'a été lancé en France le programme Carnot et, que dans les deux pays ont été jetées les bases d'un programme
... pour simplifier en les unifiant

les procédures administratives
Le PICF est le fruit d'un processus d'apprentissage 
Une fonction intégratrice et trois expériences-clés

Niveau politique et institutionnel : une démarche commune

Management et chercheurs : optimisation des complémentarités bilatéral de recherche appliquée à l'industrie, destiné aux Instituts Carnot et aux instituts membres de la Société Fraunhofer (voir REA 103/ 2011). L'objectif principal de ce programme était de permettre aux partenaires de former des alliances stratégiques orientées sur le long terme. Comme les procédures afférentes relevaient des contextes nationaux respectifs, I'harmonisation nécessaire à la réalisation de ce projet a nécessité un processus d'apprentissage notamment en matière de planification, de gestion du temps et de procédures de sélection. Sur la base des enseignements tirés de cette expérience ont alors été développés en 2008 les fondements d'un programme bilatéral de recherche commun avec une procédure commune de candidature et de sélection (Tippmann, 2013 ; Tippmann/Buoncuore, 2013 ; Zenker/Tippmann, 2011).

Ce programme vise principalement à promouvoir l'excellence scientifique, le transfert de technologies, l'orientation de la recherche sur la demande du marché, ainsi qu'à initier des coopérations stratégiques par-delà les premiers projets communs. A la différence de la plupart des autres programmes de soutien à la recherche, le programme PICF, dont la coordination est assurée par l'ANR et la Société Fraunhofer, n'est pas axé explicitement sur les technologies. Depuis sa création, trois campagnes d'appel à projets $(2008,2009,2011)$ ont été menées à bien, et les aides dans le cadre du PICF sont programmées jusqu'en 2015 (Tippmann/Buoncuore, 2013).

Dans le contexte de la recherche bilatérale, le programme PICF a une fonction intégratrice fondamentale. Les enseignements clés qu'on peut tirer au bout de cinq années d'existence sont les suivants :

- L'Agence Nationale de la Recherche (ANR) et le ministère fédéral de la Formation et de la Recherche (BMBF), qui financent ce programme, ont lancé un appel d'offres commun (en langue anglaise) destiné aux Instituts Carnot et Fraunhofer. Pour y parvenir, il a fallu un long processus de négociations et d'adaptations successives entre d'une part l'ANR et le BMBF et, d'autre part, le management du programme. Dans de nombreux domaines, les partenaires ont dû abandonner les procédures convenues pour parvenir à un consensus débouchant sur une démarche commune.

- Ainsi, il a été tenu compte des différentes manières de gérer le processus, ancrées dans les contextes et habitudes respectifs - par exemple en ce qui concerne, dans le cas français, les modalités d'implication des doctorants dans la mise en œuvre du projet (avec une programmation précise des échéances de soutenance de thèse) et, dans le cas allemand, des chercheurs (à temps plein) occupés par les instituts.

- Du fait qu'ils relèvent de contextes institutionnels nationaux différents, les instituts Carnot et Fraunhofer se distinguent par la structure de leur financement. Dans le cadre du PICF, ces particularités sont respectées. Elles constituent le seul aspect sur lequel diffèrent les candidatures respectives dans les appels à projets.

En ce qui concerne le programme lui-même, le facteur clé de succès réside dans le processus commun de son développement et de sa mise en œuvre. Seuls en effet des processus partagés et des procédures uniformes peuvent aboutir à des projets susceptibles d'être conçus et réalisés conjointement. Dans le cas du PICF, l'accord des ministres de la recherche, ainsi qu'un memorandum of understanding constituaient les fondements. Un autre facteur décisif est que l'ANR et le BMBF s'étaient mis d'accord sur une démarche commune, autrement dit, qu'ils s'étaient montrés chacun disposé à dévier des instructions et règlements en usage dans son pays au bénéfice de la définition d'un programme transfrontières harmonisé. Ainsi, c'est grâce à l'abandon d'un certain nombre de spécificités nationales qu'a pu naître un processus réellement commun.

Le management du programme a vu se développer de manière croissante des alliances stratégiques entre les partenaires. II faut dire que cette évolution avait 
été forcée par la procédure de sélection des projets de coopération, ce qui a permis de concentrer les moyens financiers sur les projets dont on estimait qu'ils avaient le potentiel pour créer des alliances stratégiques durables. Quant aux chercheurs, le programme de soutien bilatéral leur a offert la possibilité de coopérer avec des partenaires d'excellence et de mettre à profit les complémentarités, ce qui a contribué à l'excellence de leur recherche. Souvent, ce travail en commun leur a permis d'approfondir et de pérenniser leurs réseaux de contacts, tout en bénéficiant de l'accès aux réseaux du partenaire, tant dans le monde académique que dans l'industrie.

Cet APerçu des différences culturelles et des défis qui apparaissent dans les coopérations bilatérales à tous les niveaux considérés permet de dégager quelques enseignements clés susceptibles d'avoir une influence positive sur la coopération franco-allemande (et internationale) dans le domaine de la recherche.

Au niveau des personnes, c'est-à-dire des chercheurs impliqués, l'ouverture d'esprit, la curiosité face à la nouveauté comme la volonté de s'investir dans la gestion des différences culturelles sont les bases du succès d'un projet de coopération. Dans l'idéal s'y ajoutent, outre la maîtrise de la langue du partenaire et de sa culture, une connaissance approfondie de l'organisation de la recherche et des structures de son financement dans les deux pays. Au niveau institutionnel des organismes de recherche, si les 'personnes clés' jouent un rôle déterminant, le soutien apporté par les instances décisionnelles au sein des organismes de recherche, l'ancrage de ces projets dans les stratégies respectives en matière de recherche, de même que la conscience du temps qu'il faut à l'émergence d'une coopération fructueuse sont autant d'éléments décisifs pour la réussite de tels projets. Enfin, le niveau du politique comme du soutien à la recherche, où se définissent les conditions-cadre pour la coopération transfrontières, joue lui aussi un rôle clé en développant des structures favorables à la recherche et en préparant de la sorte la voie aux coopérations bi- et internationales.

La coexistence de ces trois niveaux et de leurs nombreuses interactions révèle combien le succès d'une coopération dans le domaine de la recherche est le fruit d'un permanent processus collectif d'apprentissage qui exige de chacun et à chaque niveau ouverture d'esprit, flexibilité et création de liens de confiance. Si ces trois conditions sont réunies lors de l'élaboration et de la mise en œuvre de projets transfrontières, la coopération transnationale ne permet pas seulement de réunir des compétences complémentaires et d'optimiser les ressources, elle peut également devenir un moyen de s'ouvrir sur de nouveaux partenaires et de nouveaux débouchés et apporter une contribution majeure à la stratégie d'internationalisation des organismes de recherche. Les cas types présentés ici révèlent que de nombreuses étapes ont déjà été franchies sur la voie d'alliances stratégiques orientées sur le long terme et que la voie est désormais ouverte pour mener au succès d'autres projets communs. C'est là un fort potentiel qu'il convient de développer pour améliorer encore le positionnement de la France et de l'Allemagne dans la concurrence mondiale.

(Traduction : Isabelle Bourgeois)

\section{Indications bibliographiques}

-BALlouk A., « Opening up national research programmes as a strategy for internationalising applied research », in ZeNKer A., BAIER E., MuLLER E., HÉRAUD, J.-A., TIPPMANN V. (eds), Strategies for bilateral research cooperation: French-German experiences, Fraunhofer-Verlag, Stuttgart, 2013 (2013a)

- BARMEYer C.I., DAvoINe E., «Intercultural difficulties in French-German research and development projects ». in ZenKer A., Baier E., Muller E., HÉrAUd, J.-A., TiPPMANN V. (eds), op.cit. 
-DAMIANI L., «Experiences of cooperation between Lorraine and the Saarland: The case of a cross-border business incubator », in ZeNKER A., BAIER E., MULLER E., HÉRAUd J.-A., TIPPMANN V. (eds), op. cit.

-DimRoth F., « Project-specific public funding strategies necessary for research cooperation from prototypes up to market introduction », in ZENKER A., BAIER E., MULLER E., HÉRAUD J.-A., TIPPMANN V. (eds), op. cit.

- Ebersberger B., Edler J., Frietsch R., Grimpe C., Licht G., Löhlein H., Wang J., « Ausmaß internationaler Aktivitäten in der deutschen öffentlich finanzierten Wissenschaft », in EDLER J. (ed.), Internationalisierung der deutschen Forschungs- und Wissenschaftslandschaft, Fraunhofer IRB Verlag, Stuttgart, 2007

- HAAG A., " Harmonisation of national research programmes: Enabling joint applications for research projects in basic research », in ZENKER A., BAIER E., MULLER E., HÉRAUD J.-A., TIPPMANN V. (eds), op. cit.

-JeLtSCH J.-M., « Fate of technology transfer in France: The case of the University of Strasbourg ", in Zenker A., Baier E., Muller E., HÉraud J.-A., TipPMANN V. (eds), op. cit.

- Kaufmann-Spachtholz M., de Villemagne C., Förster W., Peter H., « A French-German research institute as an example for long-term bilateral scientific cooperation », in ZENKER A., BAIER E., Muller E., HÉRAUd J.-A., TIPPMANN V. (eds), op. cit.

- Michels C., Fu J., NeuhÄUSler P., Frietsch R., «Performance and Structures of the German Science System 2012 », in Expertenkommission Forschung und Innovation (EFI), Studien zum deutschen Innovationssystem, $\mathrm{n}^{\circ}$ 6-2013

-SAVOY B., Nerlich F., « The importance of flexibility, mobility, academic freedom and cross-cultural competences for the successful organisation of bilateral research: Experiences of an ANRDFG-funded project in art history », in ZENKER A., BAIER E., MULLER E., HÉRAUD J.-A., TIPPMANN V. (eds), op. cit.

- TIPPMANN V., « An open bilateral call for applied research projects between Fraunhofer and Carnot Institutes : P2IC », in Zenker A., BAier E., Muller E., HÉrAUd J.-A., TipPMANN V. (eds), op. cit.

- TIPPMANN V., Buoncuore D., « Programme Inter Carnot-Fraunhofer (PICF) : A joint bilateral call for applied research projects between Fraunhofer and Carnot Institutes », in ZENKER A., BAIER E., Muller E., HÉRAUd J.-A., TIPPMANN V. (eds), op. cit.

-Weg-Remers S., Chelioti E., MAYER C., «French-German research collaboration in cancer research: The model of the Deutsches Krebsforschungszentrum (DKFZ) member of the Helmholtz Association and the Institut National de la Santé et de la Recherche Médicale (INSERM) in developing French-German research units », in ZENKER A., BAIER E., MULLER E., HÉRAUD J.-A., TIPPMANN V. (eds), op. cit.

-Zenker A., TIPpMAnN V., « Recherche Appliquée en RFA. Société Fraunhofer : la R\&D au service de l'industrie », Regards sur l'Economie Allemande, n 103 , hiver 2011

-Zenker A., Tippmann V., Baier E., Héraud J.-A., Muller E., Schricke E., Covillard M., « Research landscapes and innovation policies in France and in Germany: An overview » (2013b), in Zenker A., Baier E., Muller E., Héraud J.-A., Tippmann V. (eds), op. cit.

- 50 ans de coopération franco-allemande en recherche, technologie et innovation 1963-2013 / 50 Jahre Deutsch-Französische Zusammenarbeit in Forschung, Technologie und Innovation 19632013, brochure bilingue éditée par le ministère de l'Enseignement supérieur et de la Recherche et le Bundesministerium für Bildung und Forschung en coopération avec le Centre d'Information et de Recherche sur l'Allemagne contemporaine (CIRAC) et la Deutsch-Französische Gesellschaft für Wissenschaft und Technologie (DFGWT). Téléchargeable sur http://www.enseignementsuprecherche. gouv.fr/cid71488/50-ans-de-cooperation-franco-allemande-en-recherche-technologie-etinnovation.html

- http://www.enseignementsup-recherche.gouv.fr/cid71487/l-allemagne-et-la-france-fixent-de-nouveauxcaps-pour-la-cooperation-scientifique.html (« Chiffres clefs »)

-http://www.isi.fraunhofer.de/isi-de/p/publikationen/flyer/fraunhofer-carnot.php. 